\title{
QUALIDADE DA MADEIRA EM CLONE DE Eucalyptus grandis W. Hill ex Maiden SUBMETIDO A DESRAMA ARTIFICIAL ${ }^{1}$
}

\author{
Henrique Quero Polli², Geraldo Gonçalves dos Reis ${ }^{3}$, Maria das Graças Ferreira Reis ${ }^{3}$, Benedito Rocha \\ Vital $^{3}$, José Eduardo Macedo Pezzopane ${ }^{4}$ e Ivan da Costa Ilhéu Fontan ${ }^{2}$
}

\begin{abstract}
RESUMO - A qualidade da madeira para serraria foi avaliada em plantas de clone de Eucalyptus grandis, submetidas à desrama artificial, utilizando-se a combinação de diferentes alturas de remoção dos galhos, a partir do solo, e de intervenções necessárias para atingir 6 m de altura livre de galhos, em Abaeté, MG (19¹5'94" S e 4544'56" W). A primeira intervenção de desrama ocorreu quando as plantas apresentavam 16 meses de idade. A conicidade, o achatamento e o encurvamento da primeira tora não diferiram significativamente entre os tratamentos. A desrama artificial, no entanto, promoveu redução no núcleo nodoso, com um ganho médio de $94 \%$ na extensão de madeira limpa em relação à testemunha. Para ferimentos de desrama de diâmetro inferior a $1,5 \mathrm{~cm}$, a extensão de oclusão do galho foi de $0,48 \mathrm{~cm}$, com $42 \%$ dos ferimentos apresentando fenda aberta. Para ferimentos com diâmetro superior a $1,5 \mathrm{~cm}$, a extensão da oclusão foi de $0,74 \mathrm{~cm}$, com $92 \%$ dos ferimentos com fenda aberta, indicando cicatrização mais rápida em ferimentos de remoção de galhos de menor diâmetro. $\mathrm{O}$ ângulo de inclinação do galho foi mais próximo à horizontal, principalmente entre 0 e 1,5 m de altura do tronco. A densidade da madeira ao longo do tronco não foi afetada pela desrama artificial, porém decresceu do ápice até a base e aumentou da medula para a casca. Os resultados, em conjunto, indicam que a desrama artificial deve ser realizada o mais cedo possível para obtenção de maior extensão de madeira limpa nesse clone.
\end{abstract}

Palavras-chave: Desrama artificial, madeira para serraria, núcleo nodoso, madeira limpa e clone de eucalipto.

\section{EVALUATION OF SAWNWOOD QUALITY IN ARTIFICIALLY PRUNED Eucalyptus grandis W. Hill ex Maiden CLONES}

\begin{abstract}
The present study aimed at the evaluation of the influence of artificial pruning of Eucalyptus grandis Hill ex Maiden clones on wood quality for sawnboard production, in Southeastern Brazil. Branches were removed to different heights from the ground and number of interventions to reach a 6 m branch-free trunk, with the first pruning intervention starting at the age of 16 months. By the age of 55 months, no significant differences ( $p>.05)$ were found for taper, flatness and bow of the first $3 \mathrm{~m}$ log as a result of pruning. The clear wood production of the pruning treatments was, on average, $94 \%$ greater than the control. Clear wood production increased with the reduction in the diameter over occlusion (wood knotty core). The pruning wounds smaller than $1.5 \mathrm{~cm}$ in diameter showed $0.48 \mathrm{~cm}$ stub occlusion extension and the wounds larger than $1.5 \mathrm{~cm}$ showed $0.74 \mathrm{~cm}$ stub occlusion extension. The branch angle was larger for the thinner branches and had no correlation with pruning. Wood density along the trunk was not affected by artificial pruning, however, it decreased from the top to the base of the first log and increased from pith to bark. The overall results indicate mainly that the genetic material for sawnboard production must present smal diameter branches and pruning must be carried out as early as possible to attain larger clear wood extension.
\end{abstract}

Keywords: artificial pruning, sawnwood quality, wood knotty core, clear wood production and eucalypt clone.

\footnotetext{
${ }^{1}$ Recebido em 20.05.2005 e aceito para publicação em 05.04.2006.

2 Programa de Pós-Graduação em Ciência Florestal da UFV, 36570-000Viçosa - MG, Brasil.

${ }_{3}^{3}$ Departamento de Engenharia Florestal da UFV, 36570-000 Viçosa-MG, Brasil. E-mail: <greis@ ufv.br>.

${ }^{4}$ Departamento de Engenharia Rural da UFES, 29500-000 Alegre, ES.
} 


\section{INTRODUÇÃO}

No Brasil, tem crescido o interesse pelo uso de materiais genéticos do gênero Eucalyptus para a produção de madeira serrada, em razão de apresentarem características físico-mecânicas e organolépticas adequadas para uso múltiplo (DEL MENEZZI e NAHUZ, 1998). A madeira de eucalipto proveniente de florestas plantadas não manejadas para esse fim, no entanto, pode conter defeitos como bolsas de resina, nós, desvios da grã e tensão de crescimento, dentre outros (PIRES, 2000; PULROLNIK, 2002; VALE et al., 2002; ALMEIDA, 2003; MONTAGU et al., 2003). Esses defeitos afetam a aparência das peças e, também, suas propriedades mecânicas, justificando a adoção de práticas silviculturais específicas, no manejo de florestas, para reduzir tais danos.

A desrama artificial proporciona melhoria na qualidade do lenho de árvores de maior diâmetro em um povoamento florestal, principalmente produzindo madeira limpa, livre de nós, desde que ocorra cicatrização completa dos ferimentos causados pela desrama, o que irá depender do diâmetro do galho, da sua localização no tronco, do vigor da planta, da idade de aplicação e da qualidade da operação da desrama artificial (SMITH, 1962; PETRUNCIO et al., 1997; BREDENKAMP et al., 1980; PIRES, 2000; PULROLNIK, 2002; MONTAGU et al., 2003). Deve-se, portanto, elaborar um plano de manejo do povoamento, adequando-se frequiência, intensidade e idade das plantas por ocasião da aplicação da desrama artificial, de forma a facilitar a cicatrização dos ferimentos, mantendo-se o núcleo nodoso reduzido e potencializando a produção de madeira de qualidade superior para serraria.

O objetivo deste trabalho foi verificar a influência da intensidade e freqüência da desrama artificial sobre a conicidade, achatamento e encurvamento da tora; ângulo de inclinação do galho; densidade ao longo do tronco; diâmetro do núcleo nodoso; extensão da oclusão do galho; e extensão de madeira limpa, em clone de Eucalyptus grandis.

\section{MATERIAL E MÉTODOS}

O estudo foi desenvolvido num povoamento do clone 24504, de Eucalyptus grandis, estabelecido no espaçamento 3 × 3 m, na Companhia Agrícola e Florestal Santa Bárbara (CAF), no Município de Abaeté, MG, localizado na latitude $19^{\circ} 15^{\prime} 94^{\prime \prime}$ S e longitude $45^{\circ} 44^{\prime} 56^{\prime \prime} \mathrm{W}$.

O experimento foi constituído de delineamento experimental inteiramente casualizado, com seis tratamentos de desrama, combinando diferentes intensidades de desrama (altura de remoção dos galhos a partir do solo) e frequiência (número de intervenções necessárias para atingir $6 \mathrm{~m}$ de altura livre de galhos na planta), com três repetições. Foram aplicados tratamentos em que a primeira intervenção de desrama atingia $0,5 \mathrm{~m}$ de altura da planta, em razão de esse clone, usualmente, apresentar galho muito grosso até essa altura, e a altura máxima de desrama na primeira intervenção foi de $1,5 \mathrm{~m}$ a partir do solo, de modo a remover no máximo $40 \%$ da altura da copa viva das plantas. As intervenções de desrama foram realizadas aos 16, 20, 28 e 45 meses de idade, conforme mostrado no Quadro 1.

A avaliação dos parâmetros de qualidade da madeira descritos a seguir foi realizada nas toras da base das árvores, com $3 \mathrm{~m}$ de comprimento, que foram levadas ao Laboratório de Ecologia e Fisiologia Florestal da UFV.

Quadro 1 - Tratamentos de desrama (intensidade e freqüência) aplicados em plantas do clone 24504 de Eucalyptus grandis, submetidas à primeira intervenção aos 16 meses de idade, em Abaeté, MG

Table 1 - Pruning treatments applied to Eucalyptus grandis clone plants starting at the age of 16 months, in Abaeté, MG, Brazil

\begin{tabular}{|c|c|c|c|c|c|}
\hline \multirow[t]{2}{*}{ Tratamentos } & \multicolumn{4}{|c|}{ Altura Parcial de Desrama (m) } & \multirow[t]{2}{*}{ Altura Total de Desrama (m) } \\
\hline & $16^{1}$ meses & 20 meses & 28 meses & 45 meses & \\
\hline Testemunha & - & - & - & - & - \\
\hline $0,5+0,5+2,0+3,0$ & $0,5^{2}$ & $0,5^{3}$ & $2,0^{3}$ & $3,0^{3}$ & 6,0 \\
\hline $0,5+1,0+1,5+3,0$ & 0,5 & 1,0 & 1,5 & 3,0 & 6,0 \\
\hline $1,0+1,0+1,0+3,0$ & 1,0 & 1,0 & 1,0 & 3,0 & 6,0 \\
\hline $1,0+0,0+2,0+3,0$ & 1,0 & $\longrightarrow$ & 2,0 & 3,0 & 6,0 \\
\hline $1,5+0,0+1,5+3,0$ & 1,5 & - & 1,5 & 3,0 & 6,0 \\
\hline
\end{tabular}

116 meses constitue a idade em que foi realizada a primeira intervenção de desrama, ${ }^{2}$ Altura de desrama a partir do nível do solo,

${ }^{3}$ Altura de desrama a partir da altura da primeira, segunda e terceira desramas.

R. Árvore, Viçosa-MG, v.30, n.4, p.557-566, 2006 
Foram determinados a conicidade, o achatamento e o encurvamento dessa primeira tora, seguindo-se as normas brasileiras para a medição e classificação de toras de madeiras de folhosas (IBDF, 1984).

A densidade básica da madeira foi obtida através da metodologia adotada por Vale et al. (2002), em que foram retirados três discos de $5 \mathrm{~cm}$ de espessura em diferentes posições nas toras. Neste trabalho, os discos foram tirados na base, a 1,3 m e no topo. Em seguida, retirou-se uma bagueta desses discos, no sentido radial, que, posteriormente, foi dividida em três segmentos iguais, no sentido medula-casca, de forma a avaliar a variação da densidade da madeira. A densidade das amostras foi obtida segundo o método de imersão, conforme descrito por Vital (1984).

Para a avaliação da cicatrização interna dos ferimentos pós-desrama, as toras foram divididas em seis porções de $0,5 \mathrm{~m}$, realizando-se cortes transversais e longitudinais sobre os ferimentos provenientes do corte de galho, em duas classes distintas de diâmetro de ferimento ( 0 a $1,5 \mathrm{~cm}$ e maior que $1,5 \mathrm{~cm}$ ) (Figura 1). Os cortes foram executados no centro do ferimento. No corte transversal, avaliaram-se: a extensão de madeira limpa, a extensão da oclusão do galho, o diâmetro do núcleo nodoso; a existência de espaços abertos nas cicatrizes, nos sentidos radial e longitudinal; e a presença ou ausência de bolsas de resina (Figura 2). Nos cortes longitudinais, avaliaram-se a extensão de madeira limpa, o diâmetro do núcleo nodoso e o ângulo formado pela mancha do galho desramado em relação à medula (Figura 2).

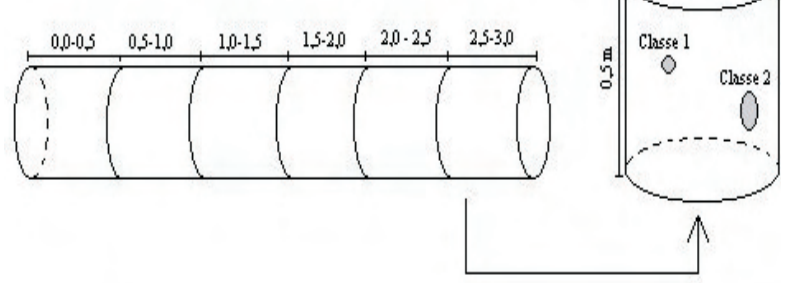

Figura 1 - Esquema de tora seccionada em seis porções de $0,5 \mathrm{~m}$ para obtenção de cortes transversais e longitudinais para análise das cicatrizes de ferimento de desrama, em Abaeté, MG.

Figure 1 -Diagram of sectioned trunk to obtain radial and longitudinal wood samples to study the pruning wound closure in the first log of Eucalyptus grandis clone, in Abaeté, MG, Brazil.

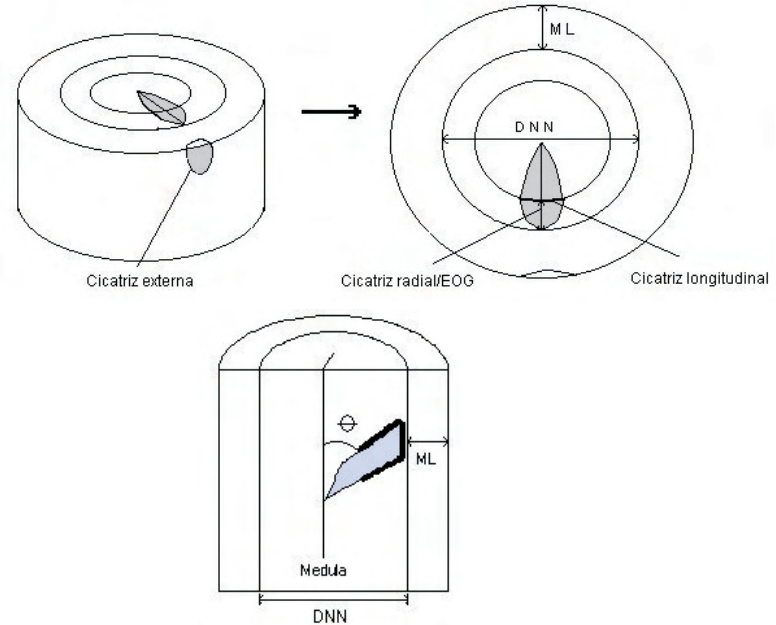

Figura 2 - Esquema do disco da tora para análise da cicatrização do ferimento da desrama em cortes transversal e longitudinal em plantas do clone 24504 de Eucalyptus grandis, em Abaeté, MG(EOG - extensão da oclusão do galho, DNN - diâmetro do núcleo nodoso, ML - extensão de madeira limpa e $\theta$ - ângulo de inclinação do galho).

Figure 2-Diagram of sectioned trunk showing the method used to obtain the extension of branch occlusion (EOG), diameter over occlusion (DNN), clear wood extension $(M L)$ and branch angle $(\theta)$, in radial and longitudinal direction in the first log of Eucalyptus grandis clone, in Abaeté, MG, Brazil.

Os dados de extensão de madeira limpa e o diâmetro do núcleo nodoso, obtidos nos dois tipos de cortes, foram analisados em conjunto; os dados de extensão da oclusão do ferimento foram analisados apenas para o corte transversal; e o ângulo formado entre o galho e a medula foi analisado para o corte longitudinal.

O efeito dos tratamentos foi avaliado com o auxílio da análise de variância. Quando os efeitos dos tratamentos foram significativos pelo teste F, realizaramse comparações de médias através do teste de Duncan a 5\% de probabilidade. Essas análises estatísticas foram executadas empregando-se o Sistema de Análise Estatística (SAEG).

\section{RESULTADOS E DISCUSSÃO}

\subsection{Conicidade, achatamento e encurvamento}

Aos 55 meses de idade, as diferentes intensidades e freqüências de desrama adotadas neste estudo não influenciaram significativamente a conicidade,

R. Árvore, Viçosa-MG, v.30, n.4, p.557-566, 2006 
achatamento e encurvamento da tora que foi submetida à primeira intervenção de desrama aos 16 meses de idade. De qualquer forma, segundo a norma brasileira de classificação de toras (IBDF, 1984), a conicidade e o encurvamento são considerados defeitos apenas quando apresentam valores superiores a $3 \%$ e, o achatamento, apenas quando atingem valor inferior a $90 \%$. Considerando que a seleção dos clones utilizados para reflorestamento é baseada na sua produtividade e qualidade do fuste, dentre outras características, é de se esperar que eles apresentem conicidade, achatamento e encurvamento baixos.

O maior valor de conicidade foi $1,18 \%$ (tratamento $0,5+1,0+1,5+3,0)$, e o menor valor foi para a testemunha (Quadro 2). Esse resultado foi semelhante ao encontrado por Vale et al. (2002), que, ao analisarem a conicidade em toras de $6 \mathrm{~m}$ de comprimento, de diferentes clones de eucalipto, com 6 anos de idade, não encontraram efeito significativo das diferentes alturas de desrama sobre a conicidade das árvores. Pires (2000) observou tendência de redução da conicidade com o aumento da intensidade de desrama em toras de Eucalyptus grandis aos 92 meses de idade. Em idades mais jovens, no clone utilizado neste estudo, Pulrolnik (2002) também não observou diferenças em função da desrama artificial quando foi analisada a conicidade da tora de $3 \mathrm{~m}$, no entanto, quando foram examinadas as seções da tora, observaram-se diferenças significativas da conicidade nas seções 1,30-2,30 m e 1,30-3,30 m de altura na tora a partir da sua base, em razão de o clone em estudo apresentar concentração de galhos grossos entre 1,30 e 2,30 $\mathrm{m}$ de altura.

O valor mínimo de achatamento foi $90,6 \%$ no tratamento $0,5+1,0+1,5+3,0$, valor próximo ao considerado defeito, de acordo com a norma brasileira de madeira serrada (IBDF, 1984). Pires (2000) não encontrou efeitos de tratamentos de desrama sobre o achatamento da tora da base de Eucalyptus grandis avaliados aos 7 anos de idade, em Dionísio, MG.

O encurvamento das toras deste estudo também em nenhum tratamento foi superior a $3 \%$ (Quadro 2), quando é considerado defeito pela norma brasileira de classificação de toras, resultado similar ao observado por Pires (2000) em E. grandis.

\section{2. Ângulo de inserção do galho}

O ângulo de inserção do galho não variou significativamente entre os tratamentos de desrama, porém foram observadas diferenças significativas entre as classes de diâmetro do ferimento e na interação entre as classes de diâmetro dos ferimentos de desrama e os tratamentos de desrama.

Analisando o ângulo de inserção do galho, por classe de diâmetro de ferimentos de desrama (Quadro 3 ), verificou-se que os galhos de maior diâmetro, que geram ferimento maior do que $1,5 \mathrm{~cm}$, tiveram o ângulo de inserção correspondente a $40,6^{\circ}$, significativamente menor do que a inclinação de $43,0^{\circ}$ observada em galhos mais finos (ferimentos com diâmetro inferior a $1,5 \mathrm{~cm}$ ). Comparando os tratamentos de desrama, verifica-se que os tratamentos $1,5+0,0+1,5+3,0 ; 0,5+1,0+1,5+3,0$ e 1,0+1,0+1,0+3,0 apresentaram ângulos de inserção dos galhos mais grossos significativamente mais elevados do que os dos demais tratamentos. Os galhos grossos mais verticais, localizados até a altura de aproximadamente $1,5 \mathrm{~m}$, foram retirados no momento da primeira ou segunda intervenção de desrama artificial nesses tratamentos; nos outros dois tratamentos foram retirados os galhos até no máximo 1,0 m de altura no tronco. De modo geral, independentemente da altura na primeira tora, os galhos finos apresentaram ângulo mais próximo da horizontal do que os galhos grossos.

\subsection{Densidade da madeira ao longo do tronco}

A densidade da madeira ao longo do tronco não diferiu significativamente entre os tratamentos de desrama. Schilling et al. (1997) também não observaram diferenças significativas entre os tratamentos de desrama

Quadro 2 - Conicidade, achatamento e encurvamento, em porcentagem, das toras de plantas do clone 24504 de Eucalyptus grandis, submetidas à primeira intervenção de desrama aos 16 meses de idade, em Abaeté, MG

Table 2-Taper, flatness and bow (\%) in the first log of Eucalyptus grandis clone, subjected to pruning starting at the age of 16 months, in Abaeté, MG, Brazil

\begin{tabular}{lccc}
\hline Tratamentos & $\begin{array}{c}\text { Conicidade } \\
(\%)\end{array}$ & $\begin{array}{c}\text { Achatamento } \\
(\%)\end{array}$ & $\begin{array}{c}\text { Encurvamento } \\
(\%)\end{array}$ \\
\hline Testemunha & $0,83 \mathrm{a}$ & $98,09 \mathrm{a}$ & $0,79 \mathrm{a}$ \\
$0,5+0,5+2,0+3,0$ & $1,04 \mathrm{a}$ & $92,70 \mathrm{a}$ & $0,72 \mathrm{a}$ \\
$0,5+1,0+1,5+3,0$ & $1,18 \mathrm{a}$ & $90,56 \mathrm{a}$ & $1,09 \mathrm{a}$ \\
$1,0+1,0+1,0+3,0$ & $1,09 \mathrm{a}$ & $97,65 \mathrm{a}$ & $1,25 \mathrm{a}$ \\
$1,0+0,0+2,0+3,0$ & $1,14 \mathrm{a}$ & $94,71 \mathrm{a}$ & $0,52 \mathrm{a}$ \\
$1,5+0,0+1,5+3,0$ & $0,97 \mathrm{a}$ & $94,52 \mathrm{a}$ & $1,30 \mathrm{a}$ \\
\hline
\end{tabular}

As médias seguidas pela mesma letra minúscula na coluna não diferem entre si, a $5 \%$ de probabilidade, pelo teste de Duncan. 
na densidade da madeira de Pinus elliottii. Vale et al. (2002) observaram que a desrama artificial promoveu redução significativa na densidade da madeira em clones de eucalipto, em comparação com a testemunha. Segundo Elliott (1970), citado por Vale et al. (2002), a desrama artificial de galhos vivos causa a redução na produção de lenho inicial na madeira formada no tronco, influenciando, assim, a densidade básica da madeira. Schilling et al. (1998), no entanto, estudando a influência de diferentes intensidades de desrama sobre a produção de lenho tardio na madeira de Pinus elliottii, não observaram diferença estatística entre os tratamentos de desrama.

A densidade da madeira aumentou significativamente no sentido medula-casca e decresceu no sentido basetopo. A densidade da madeira próximo à casca $(0,57 \mathrm{~g} /$ $\mathrm{cm}^{3}$ ) foi significativamente mais elevada do que na posição intermediária $\left(0,52 \mathrm{~g} / \mathrm{cm}^{3}\right)$ e próximo à medula $\left(0,51 \mathrm{~g} / \mathrm{cm}^{3}\right)$ e, na base da tora, a densidade foi de $0,56 \mathrm{~g} /$ $\mathrm{cm}^{3}$, diferindo significativamente da densidade à altura de $1,30 \mathrm{~m}\left(0,56 \mathrm{~g} / \mathrm{cm}^{3}\right)$ e a $3,0 \mathrm{~m}$ de altura $\left(0,52 \mathrm{~g} / \mathrm{cm}^{3}\right)$. A Figura 3 apresenta variação na densidade da madeira no sentido medula-casca, nos diferentes tratamentos de desrama. $\mathrm{O}$ aumento da densidade da madeira no sentido medula-casca foi observado, dentre outros, em E. grandis e Eucalyptus saligna (TOMAZELLO FILHO, 1985), em vários clones de eucalipto (VALE et al., 2002), e em Eucalyptus microcorys e Eucalyptus pilularis (LIMA, 1996).

Quadro 3 - Ângulo de inserção do galho $\left(^{\circ}\right)$ em função da interação entre classes de diâmetro de ferimento e tratamentos de desrama em plantas do clone 24504 de Eucalyptus grandis, em Abaeté, MG

Table 3 - Branch angle $\left(^{\circ}\right)$ as a function of the interaction between pruning wound diameter and pruning treatments of Eucalyptus grandis clone, in Abaeté, MG, Brazil

\begin{tabular}{lccccc}
\hline Tratamentos & \multicolumn{3}{c}{ Classe de diâmetro do ferimento $(\mathrm{cm})$} \\
\cline { 2 - 6 } & $0-1,5$ & & $>1,5$ & \\
\hline Testemunha & $46,8 \mathrm{ab}$ & $\mathrm{A}$ & $34,8 \mathrm{~b}$ & $\mathrm{~B}$ \\
$0,5+0,5+2,0+3,0$ & $57,3 \mathrm{a}$ & $\mathrm{A}$ & $36,2 \mathrm{~b}$ & $\mathrm{~B}$ \\
$0,5+1,0+1,5+3,0$ & $46,0 \mathrm{ab}$ & $\mathrm{A}$ & 42,0 & $\mathrm{~b}$ & $\mathrm{~A}$ \\
$1,0+1,0+1,0+3,0$ & $47,3 \mathrm{ab}$ & $\mathrm{A}$ & 40,2 & $\mathrm{~b}$ & $\mathrm{~A}$ \\
$1,0+0,0+2,0+3,0$ & 45,5 & $\mathrm{~b}$ & $\mathrm{~A}$ & $37,5 \mathrm{~b}$ & $\mathrm{~B}$ \\
$1,5+0,0+1,5+3,0$ & 44,7 & $\mathrm{~b}$ & $\mathrm{~A}$ & $52,8 \mathrm{a}$ & $\mathrm{A}$ \\
\hline
\end{tabular}

As médias seguidas pela mesma letra minúscula na coluna e maiúscula na linha não diferem entre si, a $5 \%$ de probabilidade, pelo teste de Duncan.

\subsection{Extensão de oclusão do galho}

A extensão da oclusão do galho variou significativamente entre os tratamentos de desrama e entre as classes de diâmetro do ferimento, tendo ocorrido, também, uma interação significativa entre as classes de diâmetro dos ferimentos de desrama e a altura na tora. Para essa análise, não foi considerada a testemunha, pois, nesse caso, a oclusão do galho ainda não se apresentava completa devido à presença de fragmentos de galhos mortos.

Observou-se que o tratamento $0,5+0,5+2,0+3,0$ apresentou maior extensão da oclusão do galho (Quadro 4), devido à presença de partes de galhos remanescentes da desrama natural, principalmente de galhos grossos. O efeito da presença de partes remanescentes de galhos sobre a oclusão do galho foi, também, observado por Pulrolnik (2002). Lima (2003), analisando o número de galhos vivos e mortos, na fase inicial deste experimento, encontrou, nesses tratamentos, grande quantidade de galhos mortos, principalmente até a altura de $1,5 \mathrm{~m}$, aos 28 meses de idade, desse clone.

A extensão da oclusão do galho foi significativamente mais elevada nos ferimentos provenientes de galhos de diâmetro superior a $1,5 \mathrm{~cm}$. Na classe de diâmetro de $0-1,5 \mathrm{~cm}$, a extensão da oclusão do galho foi de $0,48 \mathrm{~cm}$ e, na classe de diâmetro superior a $1,5 \mathrm{~cm}$, foi de $0,74 \mathrm{~cm}$. As diferenças entre classes de diâmetro de ferimento, no entanto, foram significativas apenas quando localizadas até a altura de $1,5 \mathrm{~m}$ na tora, tendo sido $0,45 \mathrm{~cm}$ nos ferimentos menores do que $1,5 \mathrm{~cm}$ e $0,93 \mathrm{~cm}$ nos maiores do que $1,5 \mathrm{~cm}$. E a diferença significativa na oclusão do galho em função da altura da tora ocorreu apenas nos ferimentos de maior diâmetro, tendo sido $0,56 \mathrm{~cm}$ na altura entre 1,5 e 3,0 m na tora e $0,93 \mathrm{~cm}$ até a altura de $1,5 \mathrm{~m}$. A extensão de oclusão do galho foi maior até a altura de 1,5 m na tora, porque esse clone apresenta, usualmente, um galho muito grosso na base da árvore e grande número de galhos grossos entre 1,0 e 1,5 m de altura. Pulrolnik (2002) observou que ferimentos provenientes de galhos grossos apresentam maior velocidade de cicatrização, principalmente no início do processo de cicatrização, porém notou que ferimentos provenientes da retirada de galhos finos apresentaram menor tempo de oclusão total do ferimento.

R. Árvore, Viçosa-MG, v.30, n.4, p.557-566, 2006 

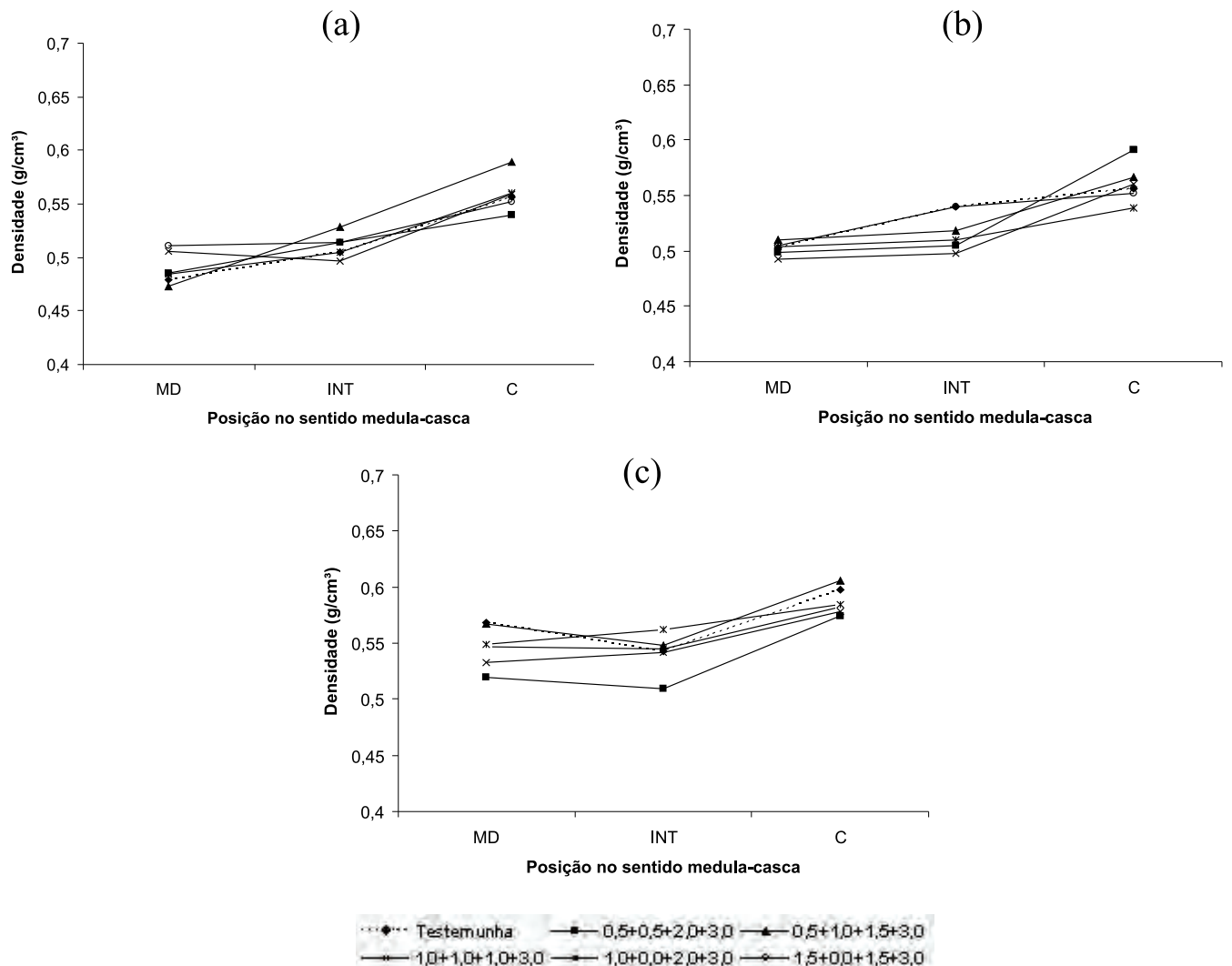

Figura 3 - Variação radial da densidade da madeira no topo (a), a 1,3 m (b) e na base (c) da primeira tora de plantas do clone 24504 de Eucalyptus grandis, em Abaeté, MG (MD = região próxima à medula, INT = região intermediária entre a medula e a casca e $\mathrm{C}=$ região próxima à casca).

Figure 3 - Wood density from pith to bark and from base to top of the first log of Eucalyptus grandis clone, in Abaeté, $M G$, Brazil $(M D=$ close to pith; INT = intermediate; $C=$ close to bark $)$.

Quadro 4 - Extensão da oclusão do galho (cm) em função dos tratamentos de desrama e das classes de diâmetro dos ferimentos de desrama em plantas do clone 24504 de Eucalyptus grandis, em Abaeté, MG

Table 4 - Extension of branch occlusion (cm) as a function of pruning treatment and pruning wound diameter in the first log of Eucalyptus grandis clone, in Abaeté, $M G$, Brazil

\begin{tabular}{cc}
\hline Tratamentos & $\begin{array}{c}\text { Extensão da oclusão } \\
\text { do galho }(\mathrm{cm})\end{array}$ \\
\hline $0,5+0,5+2,0+3,0$ & $0,98 \mathrm{a}$ \\
$0,5+1,0+1,5+3,0$ & $0,57 \mathrm{~b}$ \\
$1,0+1,0+1,0+3,0$ & $0,59 \mathrm{~b}$ \\
$1,0+0,0+2,0+3,0$ & $0,44 \mathrm{~b}$ \\
$1,5+0,0+1,5+3,0$ & $0,48 \mathrm{~b}$ \\
\hline
\end{tabular}

As médias seguidas pela mesma letra minúscula na coluna não diferem entre si, a 5\% de probabilidade, pelo teste de Duncan.

\subsection{Diâmetro do núcleo nodoso}

A análise de variância do diâmetro do núcleo nodoso apresentou diferenças significativas entre os tratamentos de desrama, classes de diâmetro do ferimento e altura na tora, tendo sido observado, também, interação significativa entre os tratamentos de desrama e classes de diâmetro dos ferimentos.

Os ferimentos localizados acima de 1,5 $\mathrm{m}$ de altura na tora apresentaram diâmetro médio de núcleo nodoso igual a $7,27 \mathrm{~cm}$, significativamente mais elevado do que para os ferimentos localizados abaixo de $1,5 \mathrm{~m}$ de altura na tora $(6,73 \mathrm{~cm})$. Até a segunda intervenção de desrama, todos os galhos até 1,5 m haviam sido removidos, exceto nos tratamentos $0,5+0,5+2,0+3,0$ e 
testemunha, ao passo que os galhos localizados acima de $1,5 \mathrm{~m}$ permaneceram por mais tempo no tronco, aumentando a extensão do núcleo nodoso.

As diferenças entre os tratamentos de desrama foram maiores na classe de ferimentos com diâmetros superiores a $1,5 \mathrm{~cm}$ do que nas classes de diâmetro inferiores a $1,5 \mathrm{~cm}$ (Quadro 5). Os tratamentos $1,5+0,0+1,5+3,0 ; 1,0+1,0+1,0+3,0 ;$ e $0,5+1,0+1,5+3,0$ não diferiram entre si e foram os que apresentaram menores valores de diâmetro do núcleo nodoso, diferenciandose significativamente dos demais tratamentos. Nesses três tratamentos, houve remoção de galhos até $1,5 \mathrm{~m}$ de altura no tronco, nas duas primeiras intervenções, enquanto nos demais tratamentos de desrama houve remoção de galhos apenas até $1 \mathrm{~m}$ de altura, nas duas primeiras intervenções. A testemunha apresentou diâmetro do núcleo nodoso, nos ferimentos de classe superior a $1,5 \mathrm{~cm}$, significativamente mais elevado do que em todos os tratamentos de desrama, devido à presença de parte de galhos mortos que foram quebrados, permanecendo externamente ao tronco. Esses resultados indicam que os galhos, principalmente os de maior diâmetro, devem ser removidos o quanto antes para se evitar redução na extensão de madeira limpa produzida.

\subsection{Extensão de madeira limpa}

A extensão de madeira limpa, produzida após a oclusão do ferimento da desrama dos galhos, variou significativamente, em nível de $5 \%$ de probabilidade, entre os tratamentos de desrama, entre as classes de

Quadro 5 - Diâmetro do núcleo nodoso $(\mathrm{cm})$ nos tratamentos de desrama em cada classe de diâmetro de ferimento de plantas do clone 24504 de Eucalyptus grandis, em Abaeté, MG

Table 5 -Diameter over occlusion (cm) as a function of the interaction between pruning wound diameter and pruning treatments of Eucalyptus grandis clone, in Abaeté, MG, Brazil

\begin{tabular}{lclc}
\hline Tratamentos & \multicolumn{3}{c}{ Classe de diâmetro do ferimento $(\mathrm{cm})$} \\
\cline { 2 - 4 } & \multicolumn{2}{c}{$0-1,5$} & $>1,5$ \\
\hline Testemunha & $6,68 \mathrm{a}$ & A & $10,93 \mathrm{a}$ B \\
$0,5+0,5+2,0+3,0$ & $6,53 \mathrm{a}$ & A & $9,00 \mathrm{~b}$ B \\
$0,5+1,0+1,5+3,0$ & $5,62 \mathrm{ab}$ A & 7,28 c B \\
$1,0+1,0+1,0+3,0$ & $6,12 \mathrm{a}$ A & 7,30 c A \\
$1,0+0,0+2,0+3,0$ & $5,50 \mathrm{ab}$ A & $9,12 \mathrm{~b}$ B \\
$1,5+0,0+1,5+3,0$ & 4,78 b A & 7,00 c B \\
\hline
\end{tabular}

As médias seguidas pela mesma letra minúscula na coluna e maiúscula na linha não diferem entre si, a $5 \%$ de probabilidade, pelo teste de Duncan. diâmetro dos ferimentos de desrama e entre as diferentes alturas da tora. Esses resultados contrastam com os encontrados por Bredenkamp et al. (1980), que não observaram diferenças significativas entre tratamentos de desrama na produção de madeira limpa em plantas de Eucalyptus grandis, na África do Sul. Esses autores observaram apenas uma tendência de aumento da porcentagem de madeira limpa produzida em árvores que receberam desrama, em relação à testemunha.

Verifica-se, no Quadro 6, que nos tratamentos com desrama artificial foi observada uma extensão média de madeira limpa da ordem de $94 \%$ mais elevada que na testemunha. A diferença na produção de madeira limpa entre a média dos tratamentos de desrama $(1,53 \mathrm{~cm})$ e a testemunha $(0,79 \mathrm{~cm})$ é de 0,74 no sentido radial, ou seja, 1,48 cm, considerando-se os dois lados opostos da tora a partir da medula. Considerando o uso de 300 árvores por hectare na produção de madeira serrada, esse aumento na produção de madeira limpa com a desrama promoverá um acréscimo de 180 tábuas de $2,5 \mathrm{~cm}$ de espessura. Considerando que a tora possui 3,0 m de comprimento e admitindo-se a produção de tábuas de $20 \mathrm{~cm}$ de largura, nos tratamentos com desrama haverá uma produção média de $2,7 \mathrm{~m}^{3} / \mathrm{ha}$ a mais de madeira serrada de alta qualidade, em relação à testemunha. Vale salientar que esse valor deve ser mais elevado quando a avaliação for realizada em idades mais avançadas, porque existem remanescentes de galhos que permaneceram no tronco de árvores sem desrama, que ainda não apresentam oclusão completa.

Quadro 6 - Extensão média de madeira limpa, em cm, em função dos tratamentos de desrama artificial em plantas do clone 24504 de Eucalyptus grandis, em Abaeté, $\mathrm{MG}$

Table 6-Clear wood extension (cm) as a function of pruning treatments in the first log of Eucalyptus grandis clone, in Abaeté, MG, Brazil

\begin{tabular}{cc}
\hline Tratamentos & $\begin{array}{c}\text { Extensão média de } \\
\text { madeira limpa }(\mathrm{cm})\end{array}$ \\
\hline Testemunha & $0,79 \mathrm{~b}$ \\
$0,5+0,5+2,0+3,0$ & $1,47 \mathrm{a}$ \\
$0,5+1,0+1,5+3,0$ & $1,51 \mathrm{a}$ \\
$1,0+1,0+1,0+3,0$ & $1,75 \mathrm{a}$ \\
$1,0+0,0+2,0+3,0$ & $1,35 \mathrm{a}$ \\
$1,5+0,0+1,5+3,0$ & $1,57 \mathrm{a}$ \\
\hline
\end{tabular}

As médias seguidas pela mesma letra minúscula na coluna não diferem entre si, a $5 \%$ de probabilidade, pelo teste de Duncan.

R. Árvore, Viçosa-MG, v.30, n.4, p.557-566, 2006 
O tratamento $1,0+1,0+1,0+3,0$ apresentou valor médio de madeira limpa de $1,75 \mathrm{~cm}$ ( $121 \%$ mais elevado que na testemunha). Essa maior extensão de madeira limpa para esse tratamento se deveu à remoção dos galhos vivos de diâmetro entre 1,0 e $2,0 \mathrm{~cm}$, que se concentravam até aproximadamente $2,0 \mathrm{~m}$ de altura na planta, conforme relatado por Lima (2003) na segunda intervenção de desrama. Pires (2000), Pulrolnik (2002) e Almeida (2003), estudando o efeito da desrama artificial sobre a produção de madeira limpa em clones de eucalipto, encontraram, também, influência positiva da desrama artificial sobre a extensão de madeira limpa. Smith (1962) e Petruncio et al. (1997) mencionaram que galhos mortos apresentam menor taxa de cicatrização do que galhos vivos, estes últimos com maior taxa de formação de calo, resultante da proliferação lateral das células do câmbio e de células parênquimáticas encontradas no limite do ferimento, o que resulta na redução da qualidade da madeira.

Os ferimentos localizados até 1,5 $\mathrm{m}$ de altura na tora apresentaram extensão de madeira limpa de $1,79 \mathrm{~cm}$, significativamente mais elevado do que nos ferimentos localizados na seção de 1,5 a 3,0 m (1,02 cm), indicando que os galhos retirados mais cedo, ou seja, até a segunda intervenção, deram origem a

(a)

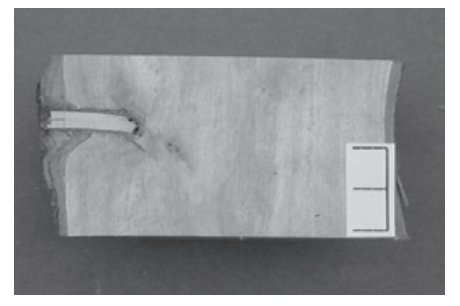

(b)

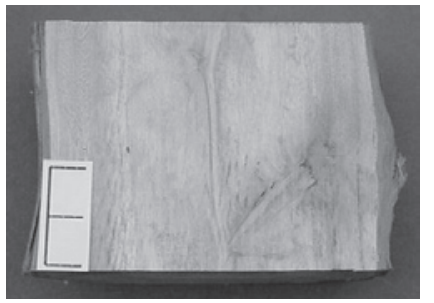

(d) ferimentos com maior taxa de cicatrização que galhos localizados acima de $1,5 \mathrm{~m}$, que permaneceram por mais tempo no tronco.

Os ferimentos de diâmetro médio maior que $1,5 \mathrm{~cm}$ apresentaram $0,78 \mathrm{~cm}$ de extensão de madeira limpa em todos os tratamentos, independentemente da posição na tora, o que foi significativamente mais baixo (137\%) do que nos ferimentos menores que $1,5 \mathrm{~cm}$, cuja extensão de madeira limpa foi de $1,76 \mathrm{~cm}$. Segundo Montagu et al. (2003), a produção de madeira limpa só ocorre após a total oclusão do ferimento, sendo possível concluir que os ferimentos maiores levam mais tempo para a total oclusão em relação aos ferimentos menores, justificando, assim, a adoção de técnicas de desrama o mais cedo possível, de forma a retirar os galhos quando estes ainda apresentarem diâmetro reduzido. Ainda não se sabe qual o diâmetro do galho que compromete a qualidade da madeira de eucalipto, sendo que alguns trabalhos indicam que galhos com diâmetros maiores que $2,5 \mathrm{~cm}$ não seriam expulsos eficientemente do tronco (MONTAGU et al., 2003). Analisando as Figuras 4 a 7, ao contrário, observa-se que galhos mesmo com diâmetro inferior a $1,5 \mathrm{~cm}$ não apresentam oclusão eficiente, em razão da permanência do galho no tronco e, se não forem removidos por desrama artificial, influenciam negativamente a extensão de madeira limpa.

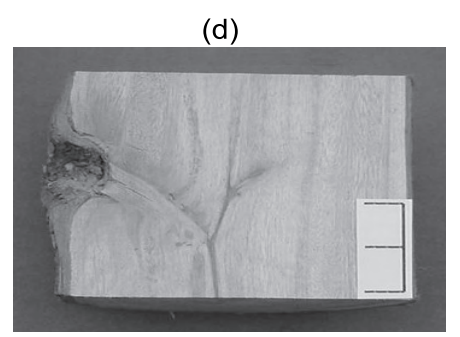

Figura 4 - Cortes longitudinais em seções do tronco para a testemunha, aos 55 meses de idade, de ferimentos da desrama natural com diâmetro (a;b) entre $0-1,5 \mathrm{~cm}$ (Classe 1) e $(\mathrm{c} ; \mathrm{d})>1,5 \mathrm{~cm}$ (Classe 2) na primeira tora de plantas do clone 24504 de Eucalyptus grandis, em Abaeté, MG (escala $=2 \mathrm{~cm})$.

Figure 4-Longitudinal sections, at the age of 55 months, for natural pruning wound diameter of (a;b) $0-1.5 \mathrm{~cm}$ (Class1) and $(c ; d)>1,5 \mathrm{~cm}$ (Class 2) in the first log of Eucalyptus grandis clone in Abaeté, MG, Brazil. 
(a)

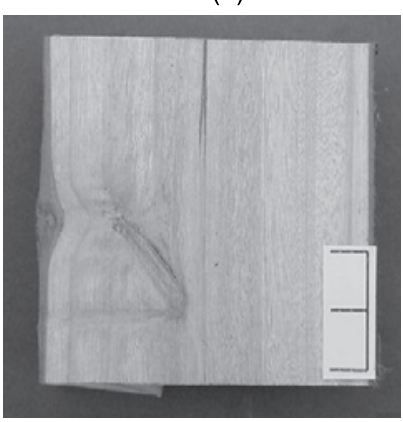

(b)

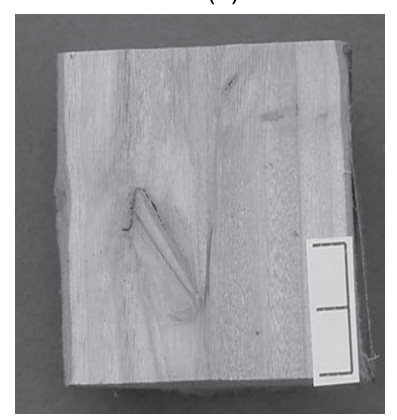

(c)

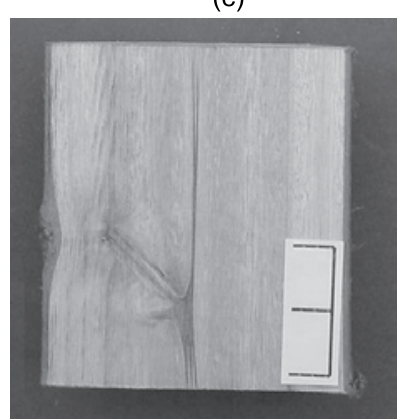

(d)

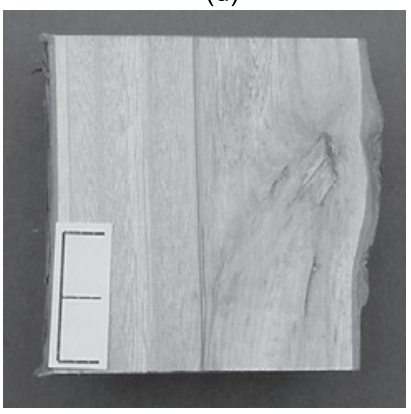

Figura 5 - Cortes longitudinais em seções do tronco para os tratamentos 1,0+1,0+1,0+3,0 (a,b) e 0,5+1,0+1,5+3,0 (c,d), aos 55 meses de idade, para ferimentos de desrama artificial com diâmetro $(\mathrm{a} ; \mathrm{c})$ entre $0-1,5 \mathrm{~m}$ (Classe 1$)$ e $(\mathrm{b} ; \mathrm{d})>1,5 \mathrm{~cm}$ (Classe 2) na primeira tora de plantas do clone 24504 de Eucalyptus grandis, em Abaeté, MG (escala $=2 \mathrm{~cm}$ ).

Figure 5 -Longitudinal sections for the treatments 1,0+1,0+1,0+3,0 (a,b) e 0,5+1,0+1,5+3,0 (c,d), at the age of 55 months, for pruning wound diameter of $(a ; c) 0-1.5 \mathrm{~cm}$ (Class 1$)$ and $(b ; d)>1.5 \mathrm{~cm}$ (Class 2$)$ in the first log of Eucalyptus grandis clone in Abaeté, MG, Brazil.

(a)

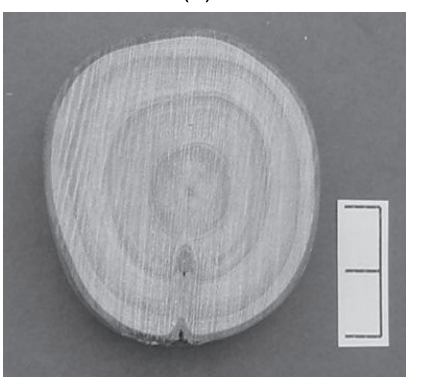

(b)

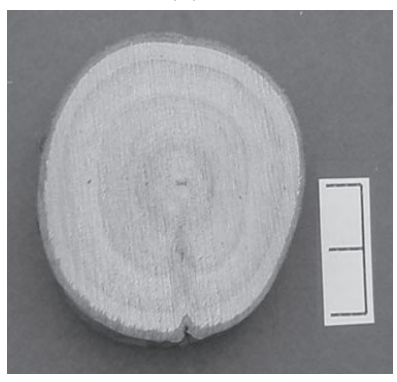

(c)

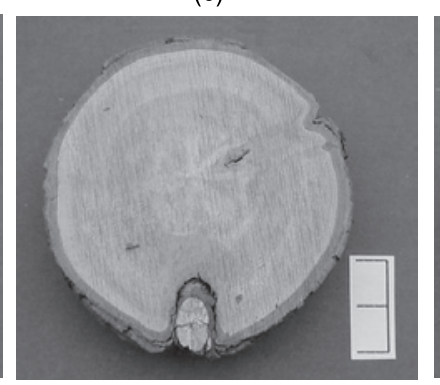

(d)

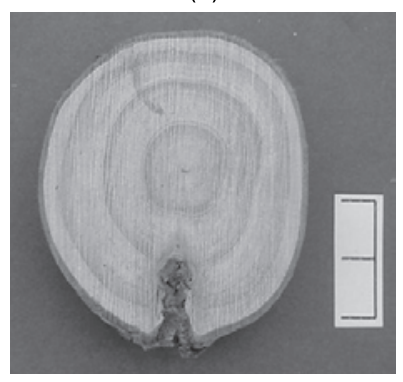

Figura 6 - Cortes transversais em seções do tronco para a testemunha, aos 55 meses de idade, de ferimentos da desrama natural com diâmetro $(\mathrm{a} ; \mathrm{b})$ entre $0-1,5 \mathrm{~m}$ (Classe 1) e $(\mathrm{c} ; \mathrm{d})>1,5 \mathrm{~cm}$ (Classe 2) na primeira tora de plantas do clone 24504 de Eucalyptus grandis, em Abaeté, MG (escala $=2 \mathrm{~cm})$.

Figure 6 - Radial sections for the control, at the age of 55 months, for natural pruning wound diameter of $(a ; b) 0-1.5 \mathrm{~cm}$ (Class1) and $(c ; d)>1.5 \mathrm{~cm}$ (Class 2) in the first log of Eucalyptus grandis clone in Abaeté, MG, Brazil.

\section{CONCLUSÕES}

A conicidade, o achatamento e o encurvamento não foram afetados significativamente pelos tratamentos de desrama artificial e não foram considerados defeitos na primeira tora de $3 \mathrm{~m}$, aos 55 meses de idade. Presumindo-se que a seleção de material genético para produção de madeira serrada leve em conta a forma da árvore, a avaliação desses parâmetros poderia não ser necessária em trabalhos futuros.

A densidade da madeira ao longo do tronco e o ângulo de inclinação dos galhos não foram afetados pela desrama artificial, não sendo, dessa forma, mais indicados para avaliar a influência da desrama artificial na qualidade da madeira para serraria.

Considerando a média de todos os tratamentos de desrama artificial, houve ganho médio de 93,7\% de madeira limpa em relação à testemunha, equivalendo a uma produção média de $2,7 \mathrm{~m}^{3} /$ ha a mais de madeira serrada de alta qualidade até a idade de 55 meses, agregando maior valor ao produto final. Isso justifica a desrama artificial como forma de melhoria na qualidade da madeira para serraria, embora seja considerada uma técnica de elevado custo.

Maior extensão de madeira limpa, menores valores de núcleo nodoso e menor extensão da oclusão dos galhos foram observados em ferimentos provocados

R. Árvore, Viçosa-MG, v.30, n.4, p.557-566, 2006 
pela remoção de galhos de menor diâmetro, indicando que o galho deve ser removido o mais cedo possível, enquanto o seu diâmetro ainda é reduzido. Esses resultados indicam, também, a necessidade de seleção de material genético que apresenta galhos mais finos até a altura da tora a ser aproveitada para serraria.

\section{AGRADECIMENTOS}

À CAF Santa Bárbara LTDA., pelo apoio financeiro e logístico oferecidos, e ao CNPq e à FAPEMIG pela concessão de bolsas e pelo apoio financeiro para a manutenção do Laboratório de Ecologia e Fisiologia Florestal da UFV.

\section{REFERÊNCIAS BIBLIOGRÁFICAS}

ALMEIDA, M.L. Desrama artificial em clones de Eucalyptus grandis $\mathbf{x}$. urophylla com diferentes arquiteturas de copa. 2003. 119f. Dissertação (Mestrado em Ciência Florestal) - Universidade Federal de Viçosa, Viçosa, 2003.

BREDENKAMP, B.V.; MALAN, F.S.; CONRADIE, W.E. Some effects of pruning on growth and timber quality of Eucalyptus grandis in Zululand. South African Forestry Journal, v. 114, p. 29-34, 1980.

DEL MENEZZI, C.H.S.; NAHUZ, M.A.R. Comportamento de Eucalyptus grandis [Hill ex Maiden] no desdobro. Revista Árvore, v. 22, n. 4, p.563-571, 1998.

\section{INSTITUTO BRASILEIRO DE DESENVOLVIMENTO FLORESTAL-IBDF. Normas para medição e classificação de toras de madeiras de folhosas. Brasília: 1984, 54p.}

LIMA, A.P.L. Aplicação de desrama em clone de Eucalyptus grandis em diferentes épocas e intensidade: efeitos sobre o crescimento e dinâmica de copa. 2003. 195f .Tese (Doutorado em Ciência Florestal) Universidade Federal de Viçosa, Viçosa, 2003.

MONTAGU, K.D.; KEARNEY, D.E.; SMITH, R.GB. The biology and silviculture of pruning planted eucalypts for clear wood production - a review. Forest Ecology and Management, n.179, p.1-13, 2003.
PETRUNCIO, M.; BRINGS, D.; BARBOUR, R.J. Predicting pruned branch stub occlusion in young coastal Douglas-fir. Canadian Journal of Forestry, v. 27, p. 1074-1082, 1997.

PIRES, B.M. Efeito da desrama artificial no crescimento e qualidade da madeira de Eucalyptus grandis para serraria e fabricação de móveis. 2000. 96f. Dissertação (Mestrado em Ciência Florestal) Universidade Federal de Viçosa, Viçosa, 2000.

PUlRolniK, K. Crescimento, dinâmica de copa e qualidade da madeira para serraria de clone de Eucalyptus grandis [Hill ex Maiden] submetido à desrama artificial. 2002. 96f. Dissertação (Mestrado em Ciência Florestal) - Universidade Federal de Viçosa, Viçosa, 2002.

SCHILLING, A.C. et al. Influência da desrama sobre a densidade da madeira de primeiro desbaste de Pinus elliottii Engelman. Revista Ciência Florestal, v. 7, n. 1, p. 77-89, 1997.

SCHILLING, A.C. et al. Influência de diferentes intensidades de desrama sobre a porcentagem de lenho tardio e quantidade de nós da madeira de primeiro desbaste de Pinus elliottii Engelman. Revista Ciência Florestal, v. 8, n. 1, p. 115-127, 1998.

SMITH, D.M. The practice of silviculture. New York: John Wiley \& Sons, 1962. 578p.

TOMAZELLO FILHO, M. Variação radial da densidade básica e da estrutura anatômica da madeira de Eucalyptus saligna e Eucalyptus grandis. IPEF, n. 29, p. 37-44. 1985

VALE, R. S. et al. Efeito da desrama artificial na qualidade da madeira de clones de eucalipto em sistema agrossilvipastoril. Revista Árvore, v. 26, n. 3, p. 285-297, 2002.

VITAL, B.R. Métodos de determinação da densidade da madeira. Viçosa, MG: SIF, 1984. (Boletim Técnico, 1). 